\title{
Die postmigrantische Perspektive: Aushandlungsprozesse in pluralen Gesellschaften
}

\author{
Naika Foroutan
}

\section{Das Postmigrantische: Eine Hinführung}

»Es ist an der Zeit, das Verhältnis zwischen Migration, Gesellschaft und Vielheit neu zu denken und den Blick auf [...] Lebenswirklichkeiten zu richten, in denen Migration zum Ausgangspunkt des Denkens wird.«, so die Einführung in die Idee des vorliegenden Sammelbandes der Kollegen Y1ldı und Hill. Auch das postmigrantische Paradigma macht - trotz terminologischer Kontraintuition - Migration zum Ausgangspunkt des Denkens. Das trügerische Präfix »post« will keineswegs einen Prozess der beendeten Migration ankündigen, im Gegenteil: Indem das »Post« den Akt der Migration zum Ausgangspunkt der Analyseperspektive erklärt, der alle Bereiche des gesellschaftlichen Lebens berührt, eröffnet es auch die Perspektive, über den Migrationsmoment hinauszublicken und gesellschaftliche Transformation in Bezug auf Aushandlungen, die mit diesem empirischen, narrativen und diskursiven Akt einhergehen, in den Fokus zu nehmen. Migration wird vom migrantisierten Subjekt als zentralem Bezugspunkt auf die gesamtgesellschaftliche Analyseebene ausgeweitet. Postmigrantisch steht also nicht für einen Prozess der beendeten Migration, sondern für eine Analyseperspektive, die sich mit gesellschaftlichen Konflikten, Narrativen, Identitätspolitiken sowie sozialen und politischen Transformationen auseinandersetzt, die nach erfolgter Migration einsetzen, und die über die gesellschaftlich etablierte Trennlinie zwischen MigrantInnen und NichtmigrantInnen hinaus Gesellschaftsbezüge neu erforscht.

Wie andere >Post<-Begriffe - wie zum Beispiel im Falle des Postkolonialismus - knüpft die postmigrantische Analyse an Kontinuitäten der Ungleichheit an und fordert, mit etablierten rassistischen Zuweisungen $\mathrm{zu}$ brechen. Das >Migrantische< im postmigrantischen Begriff steht hierbei als Chiffre für reale und konstruierte, soziale und symbolische Ungleichheiten, deren Über- 
windung sich die plurale und demokratische Einwanderungsgesellschaft zum Ziel setzt. Terminologisch wäre es wohl sinnvoller, von Post-Migrantisierung zu sprechen, um die normative Zielsetzung des Begriffes zu erfassen. Da der Begriff jedoch in der Kunst- und Kulturszene rund um das Berliner Off-Theater Ballhaus Naunynstrasse von Shermin Langhoff als subversiver und antirassistischer Begriff der Neuaushandlung von kategorialen Zuschreibungen etabliert wurde (Langhoff 2011), wird auf die terminologische Kraft des Begriffes in Zusammenhang mit seinem Entstehungskontext vertraut.

\section{Operationalisierung des Postmigrantischen: DREI ZUGÄNGE}

Der Begriff der postmigrantischen Gesellschaft kann gleichzeitig empirischanalytisch, gesellschaftspolitisch und normativ gelesen werden. Der empirische Zugang ist wohl am ehesten für eine Operationalisierung in den Sozialwissenschaften geeignet. Das »Post« in postmigrantisch steht in diesem empirischen Zugang für den Moment nach der Migration, also für Postmigration. Wir fragen: Wie verändern sich Gesellschaften, nachdem Migration erfolgt ist? Der gesellschaftspolitische Zugang ist komplizierter. »Post« meint hier eher ein Dahinter. Analytisch geht es darum, zu erkennen, wie die Omnipräsenz des Themas Migration - diese regelrechte Obsession, die mit ihr einhergeht, wie Riem Spielhaus sagt (Spielhaus 2012) - die Gesellschaft vor sich hertreibt und zentrale zugrundeliegende Konflikte überdeckt. Auf der normativen Ebene gilt es, einen moral-philosophischen Ansatz hervorzuheben. Dieser schlägt sich in der Aufforderung nieder, etablierte Prozesse des Ausschlusses und des Othering sichtbar zu machen.

\section{Der empirisch-analytische Zugang}

Für eine empirische Analyse der deutschen, postmigrantischen Gesellschaft stellt sich vorrangig die Frage: Wie verändert sich das Land, seitdem und nachdem Migration als konstituierender Bestandteil der Selbstdefinition (»Deutschland ist ein Einwanderungsland «) politisch anerkannt wurde? Erst nach der politischen Anerkennung werden rechtliche und gesellschaftliche Aushandlungen zu diesem Thema im Diskurs als gesetzlich legitim anerkannt.

Um die Einstellung der Bevölkerung zu postmigrantischen Auseinandersetzungen in Deutschland zu erforschen, haben wir am Berliner Institut für empirische Integrations- und Migrationsforschung (BIM) im Rahmen einer Erhebung die Bevölkerung in Deutschland zu folgenden Aspekten befragt (Foroutan et al. 2014): 
I. Pluralitätsbezüge: Wie ist die Einstellung zu Differenz und zu Pluralisierungsprozessen, die sich durch und nach der Migration einstellen - sprich Einstellungen zu kultureller, ethnischer, religiöser und nationaler Vielfalt, die durch und nach Migrationsbewegungen entstehen? Welche Öffnungsund Schließungsmuster sind gegenüber religiösen Minderheiten gegeben? Welche soziale Nähe wird hergestellt und wie manifestiert sich Distanz?

2. Nationalbezüge: Welche nationalen Narrative sind dominant? Wie wird eine kollektive Identität in einer Gesellschaft, die von Migration geprägt ist, definiert? Welche Narrative mit Bezug auf Vergangenheit, Normen und Werte und Zugehörigkeiten gibt es? Wer gehört in der Wahrnehmung der Bevölkerung zu dieser Gesellschaft, sozusagen zum nationalen Narrativ dazu und wer nicht? Wer wird als deutsch gesehen und wer nicht? Zählen migrantische Identitäten zum nationalen Wir? Wie stark ist die nationale Verbundenheit und was für einen Effekt hat das auf Pluralitätsakzeptanz?

3. Religionsbezüge: Wie sind die Einstellungen gegenüber demokratisch legitimen Rechten von Minderheiten (in dem Fall von Musliminnen und Muslimen) auf Partizipation, Sichtbarkeit und Gleichbehandlung? Welche Rechte werden ihnen zugestanden, welche verwehrt? Wird kulturell-religiöse Entfaltungsmöglichkeit gewährleistet und wertgeschätzt oder als Bedrohung empfunden und abgewertet? Welche räumliche Präsenz wird als legitim betrachtet und wo ist sichtbare Präsenz umstritten?

4. Wissens- und Kontaktbezüge: Wie hoch schätzt die Bevölkerung ihr Wissen über Musliminnen und Muslime ein? Woher beziehen die Bürgerinnen und Bürger ihr Wissen? Sehen sie Musliminnen und Muslime als Teil der Gesellschaft? Gibt es Nähe-Kontakte? Besteht das Wissen in stereotypem Wissen?

Auch Fragen nach institutionellem Wandel von Ministeriumszuschnitten, Bildungsinstitutionen, Arbeitsmarkt, Gesundheitssystem, Parteien etc. können empirisch mit Bezug auf Postmigration beforscht werden, außerdem natürlich Gesetzgebung, Diskurse und Narrative.

Die Studien der Reihe »Deutschland postmigrantisch « ${ }^{1}$ konzentrierten sich bei der Auswertung der Ergebnisse vorrangig auf die Einstellung der Bevölkerung gegenüber Musliminnen und Muslimen. Die leitende Annahme war, dass die Akzeptanz einer pluralen, vielfältigen, postmigrantischen Gesellschaft als neuer deutscher Lebensrealität sich am Umgang mit und der Einstellung zu kulturellen, ethnischen, religiösen oder nationalen Minderheiten messen lässt.

1 | Die bisher erschienenen Studien der Reihe "Deutschland postmigrantisch" sind auf folgender Projektseite einsehbar: https://www.projekte.hu-berlin.de/de/junited/ studien 


\section{Der gesellschaftspolitische Zugang}

Die Kernfrage, die sich für einen gesellschaftspolitischen Forschungszugang stellt, ist die nach Konfliktformationen und ihren diskursiven Grundlagen: Läuft die gesellschaftliche Bruchlinie wirklich entlang der Kategorie »Migration «? Die Konzentration des gesellschaftspolitischen Diskurses auf die Frage der Zugehörigkeit des Islam und der MuslimInnen - vor allem markiert als MigrantInnen (Spielhaus 2011) - zu Deutschland kann auch als konfliktiver Aushandlungsraum von Pluralität und Heterogenität gedeutet werden. Wenn wir genauer hinschauen, stellen wir fest, dass eine derzeit zentrale Bruchlinie zwischen PluralitätsbefürworterInnen und PluralitätsgegnerInnen verläuft. Wird Pluralität ertragen, akzeptiert und befürwortet oder verängstigt sie und ruft Widerstand hervor? Das scheint der dynamische Konfliktkern in der postmigrantischen Gesellschaft zu sein, der die Gesellschaft um zwei Pole gruppiert. Migration ist dabei nur eine Chiffre für Pluralität, hinter der sich vieles versteckt: Umgang mit Gender-Fragen, Religion, sexueller Selbstbestimmung, Rassismus, Schicht und Klasse, zunehmende Ambiguität und Unübersichtlichkeit.

Europa, Eliten, Geflüchtete und Minderheiten, besonders Muslime - alle Angriffspole der RechtspopulistInnen stehen sinnbildlich für Pluralität: Europa als pluraler Zusammenschluss von Nationen, Eliten als entfremdete Kosmopoliten einer globalen Pluralität und Geflüchtete, MigrantInnen, MuslimInnen sowie andere Minderheiten als die Ordnung bedrohende amorphe Masse der Heterogenität. Pluralisierung entgrenzt bisher vermeintlich klar Abgezirkeltes. Und was macht das Überwinden von Grenzen deutlicher als Migration? Pluralität hat es schon immer gegeben. Dass sie sich physisch manifestiert, geschieht jedoch auch über Menschen, die anders aussehen, andere Sprachen sprechen. Migration überlagert in der Argumentation der RechtspopulistInnen alle anderen, auch abgelehnten Pluralitätskategorien - Anti-Europa, AntiGender, Anti-LGBTQ, Anti-Elite -, weil es eine materielle Manifestation für das Überwinden von Grenzen darstellt. Pegida \& Co wollen wieder in ihre klar abgezirkelten Grenzen zurück, und das ist territorial, symbolisch und narrativ zu verstehen.

Entlang dieser Konfliktkategorie - Haltung zu Pluralität - bilden sich parallel jedoch neue Allianzen. Ein Dompropst macht das Licht aus, weil Pegida vor seiner Kirche demonstriert und vor seiner Kirche marschieren dann auf der Nopegida-Demo Antifa-AktivistInnen und VertreterInnen von Migrantenorganisationen neben dem herkunftsdeutschen evangelischen Lehrer und der katholischen Heimatvertriebenen mit einem Schild »Deutschland ist bunt«! Im anderen Lager, den Antagonisten, die Pluralität ablehnen - von stark bis latent - finden wir auch ein sehr heterogenes Feld: Nicht nur Rechtsextreme und Salafisten, sondern auch Mittelschicht und »besorgte Bürger« sowie Teile 
etablierter Eliten. Diese neuen Feldorientierungen, die sich teilweise außerhalb etablierter Feldzuschreibungen aufstellen, führen zu weiter zunehmenden Irritationen und Ambivalenzen. Es entsteht das Gefühl, nichts ist mehr so, wie es immer war.

Diese beiden polaren Lager bilden sich offenbar jenseits von Rechts und Links, jenseits von Alt und Jung oder von Reich und Arm, jenseits von migrantisch und nichtmigrantisch. Es ist eben nicht so, dass die Armen gegen Migration sind und die Reichen, weil sie es sich leisten können, pluralitätsaffin wären. 55 Prozent der AfD-Wähler haben Abitur, 44 Prozent verdienen über 3000 Euro und die meisten arbeiten als Angestellte (Nienhaus 2015). Den Diskurs so zu führen, als sei die Pluralitätsabwehr ein Makel der Armen und Ungebildeten, verdeckt den Rassismus der Etablierten. Es ist auch nicht so, dass MigrantInnen alle für Pluralität sind und NichtmigrantInnen dagegen. Viele MigrantInnen der ersten Generation haben sich abwehrend gegen die Willkommenskultur geäußert. Es geht, wie bereits beschrieben, um die Akzeptanz oder Abwehr von zunehmender Pluralität. Und zwischen diesen beiden Polen findet ein dynamischer Kreislauf um Anerkennung, Partizipation sowie Gleichheits- und Zugehörigkeitsprozesse statt. Dieser dynamische Konfliktzirkel mit seinen Gleichzeitigkeiten ist es, der die postmigrantische Gesellschaft antreibt und die derzeitige Situation so extrem ambivalent erscheinen lässt.

Diese gesellschaftspolitische postmigrantische Perspektive soll vor allem eine Dekonstruktionsleistung erbringen und etablierte Vorannahmen in Frage stellen oder neu reflektieren. Selbstverständlich werden die empirisch-analytische und die gesellschaftspolitische Perspektive für die Beforschung der postmigrantischen Gesellschaft zusammengedacht.

\section{Der normative Zugang}

Im normativen Zugang werden diskursive und narrative Deutungsverschiebungen ausgearbeitet, welche auf die gesamte Gesellschaft und ihre dynamische Auseinandersetzung mit Migration abzielen. Der normative Zugang fordert hierbei eine radikale, antirassistische Ausweitung der Perspektive auf Migration und eine Auseinandersetzung mit gesellschaftspolitischen Konflikten um symbolische und materielle Anerkennung, die MigrantInnen und ihren Nachkommen verwehrt bleibt. Dabei geht es auch um die Frage der Privilegiensicherung hegemonialer Akteure und um die Überwindung der etablierten Trennlinie zwischen MigrantInnen und NichtmigrantInnen.

Eine Gesellschaftsbeschreibung, die eine postmigrantische Analyseperspektive einnimmt, geht damit nicht mehr von einer natürlichen Unterscheidung zwischen Deutschen und MigrantInnen aus, sondern hinterfragt zugewiesene Positionen von Etablierten und Hinzugekommenen. Ziel ist, ein Gesellschaftsnarrativ zu entwickeln, das sich nicht in binären Kategorien von 
migrantisch versus einheimisch erzählt. Shermin Langhoff hat die postmigrantische Perspektive beschrieben als "gemeinsamen Raum der Diversität jenseits von Herkunft« (Langhoff 2011).

\section{Die Aushandlung der Pluralen Demokratie}

Postmigrantische Aushandlungsprozesse drehen sich um die Verwirklichung des Versprechens der Einwanderungsgesellschaft: Gleichheit für alle, unabhängig von sexueller Orientierung, Geschlecht, Alter, Religion, Hautfarbe oder Herkunft. Minderheitenrechte und -positionen werden in postmigrantischen Gesellschaften offensiver ausgehandelt und Fragen nach nationaler Identität, Zugehörigkeit, Repräsentation und Privilegien neu gestellt. Zu den zentralen Etappen auf dem Weg zur normativen und sinnstiftenden Zielsetzung der postmigrantischen Gesellschaft werden gezählt: 1) Die Anerkennung von symbolischer und materieller Zugehörigkeit, die die politische Anerkennung, Einwanderungsland geworden zu sein, miteinschließt. 2) die Aushandlung von Rechten, deren Verwehrung als konträr zum demokratischen Gleichheitsanspruch betrachtet wird; 3) die Etablierung antagonistischer Positionen im gesellschaftspolitischen Spektrum, die in Form neuer rechter Bewegungen, Pluralität und Hybridität klar ablehnen; 4) die Formierung von Allianzen, die auf der Grundlage einer geteilten Haltung innerhalb der Zivilgesellschaft als Gegenpol zu antagonistischen Positionen für eine plurale Demokratie eintreten; und 5) das Sichtbarwerden von Ambivalenzen und Ambiguitäten, die insbesondere die Fähigkeit einer Gesellschaft herausfordern, mit Mehrdeutigkeiten ohne negative Abwertung umzugehen.

Die postmigrantische Gesellschaftsanalyse beschreibt somit fünf zentrale Prozesse, welche die Dynamik der Transformation von Gesellschaften bestimmen und hier skizzenhaft erläutert werden sollen:

I. Anerkennung: Postmigrantische Gesellschaften entstehen, wenn die Tatsache, ein Einwanderungsland geworden zu sein, Eingang in das dominante Narrativ einer Gesellschaft findet. In Deutschland erfolgte die offizielle Anerkennung im Jahr 2001 (Unabhängige Kommission »Zuwanderung« 2001: 1), in Kanada in den 1970er Jahren und in den USA in den 1960 er Jahren. Diese Entwicklungen führten zu rechtlichen Veränderungen und zu Neudefinitionen symbolischer und materieller Zugehörigkeit, die MigrantInnen und ihren Nachkommen einen gleichen Anspruch zugestanden und sie zu legitimen Stakeholdern in der Gesellschaft machten. Erst durch diese Prozesse wurde der Weg zu einer pluralen Demokratie basierend auf dem demokratischen Gleichheitsanspruch geebnet, der die legitime Aushandlung von Positionen, Sichtbarkeit und Privilegien für alle Menschen 
eröffnet. Nichts desto weniger handelt es sich bei diesen Aushandlungen um keine neuen Phänomene. Zwar kann die offizielle Anerkennung in Deutschland im Jahr 2001 durchaus als ein narrativer Turn gesehen werden, infolgedessen die Kämpfe um Rechte und Privilegien rechtlich und moralisch zwar legitimiert wurden, die Anerkennung aber keineswegs den Startpunkt der Kämpfe darstellt. Im Gegenteil: Die vorangegangenen migrantischen Kämpfe waren nicht zuletzt der Katalysator für das Bekenntnis, ein Einwanderungsland geworden zu sein. Was die Kämpfe nach erfolgter Ankerkennung von jenen vor der offiziellen Erklärung unterscheidet, bezieht sich zum einen auf die diskursive Konstruktion einer Legitimität der migrantischen Realität, die faktisch schon längst existierte, und zum anderen auf den materiellen und rechtlichen Anspruch von Minderheiten auf Gleichheit, der es erlaubt, bestehende Ungleichheiten in Frage zu stellen. Die Kämpfe um gleiche Rechte sind damit sowohl Vorläufer als auch ein konstitutives Element postmigrantischer Gesellschaften.

2. Aushandlung: Die aktive Aushandlung von Rechten und Privilegien geht immer auch mit gesellschaftlichen Konflikten einher, die durch die Diskrepanz zwischen Akzeptanz und Ablehnung der gestellten Forderungen auftreten: MigrantInnen und ihre Nachkommen verlangen mehr repräsentative, sichtbare Positionen in Politik, Kultur, Sport, öffentlichen Räumen etc., wobei die Privilegien der Mehrheitsgesellschaft in Frage gestellt werden. Konfliktive Aushandlungen führen zunehmend zur Positionierung innerhalb von Gesellschaften und zur Herausbildung von Pro- und Anti-Diversitätsidentitäten. Die bisher hierarchisch konstruierten Konzepte von Werten und Normen werden durch zunehmende Diversität und vor allem durch Hybridität herausgefordert. Zeitgleich wird aufgrund der sich radikalisierenden Gegenpositionen der Ruf nach Antidiskriminierungsgesetzen und anderen Maßnahmen zum Schutz von Minderheiten und zum Schutz der pluralen Demokratie lauter. Innerhalb dieses Konfliktes eröffnet sich aber auch die Chance, neue, rivalisierende diskursive Hegemonien zu gestalten, die auf der Grundlage des Versprechens der Gleichheit basieren und damit auf moralischer Ebene den Diskurs antreiben. Der Kampf um die Etablierung des Gleichheitsanspruches aber muss stets als ein Kampf ohne sicheren Ausgang betrachtet werden, in welchem Minderheiten etablierte Strukturen grundlegend in Frage stellen (Spivak 1988).

3. Ambivalenzen und Ambiguitäten: Die Obsession mit Phänomenen der Migration als allgegenwärtiges und dominantes Thema in Einwanderungsgesellschaften führt - zugespitzt formuliert - zu zwei gegensätzlichen Reaktionen: Einerseits wird Migration als »natürlicher« Aspekt zeitgenössischer moderner Gesellschaften in einer globalisierten Welt verstanden und die migrantische Realität aktiv anerkannt; andererseits begünstigt der Mangel an antirassistischer politischer Bildung die sich verfestigende Konst- 
ruktion hegemonialer Machtverhältnisse zu Gunsten etablierter Gruppen (Elias/Scotson 2002: 7) und produziert Ängste vor Infiltration und dem Verlust der eigenen - vermeintlich homogenen - Kultur durch Migration. Die Diskrepanz zwischen affektiver, emotionaler Ablehnung und gleichzeitiger kognitiver Akzeptanz, die sich aus einem normativen und moralischen Wissen speist, erzeugt eine verunsichernde Dissonanz (Foroutan/ Canan 2016).

Dieses normative Paradoxon wird in Deutschland vor allem in der Einstellung der Bevölkerung zur muslimischen Minderheit deutlich (ebd.: 164): Fundamentale demokratische Grundrechte, deren allgemeiner Geltung sich die Gesellschaft zwar bewusst ist, werden dabei auf der Grundlage individueller, emotionaler Erfahrung angegriffen und hinterfragt. Eine Studie des Berliner Instituts für empirische Integrations- und Migrationsforschung zeigte, dass fast 70 Prozent der Befragten der Meinung waren, dass Muslime dazu berechtigt seien, kulturelle, religiöse und soziale Forderungen zu stellen. Gleichzeitig würden 6o Prozent der Befragten die Beschneidung junger muslimischer Männer verbieten, 50 Prozent wollten nicht, dass Lehrerinnen mit Kopftuch unterrichten und 40 Prozent der Befragten sprachen sich für die Einschränkung des Moscheebaus in Deutschland aus (Foroutan et al. 2014: 35ff.). Obwohl das Wissen über die Legitimität des Grundrechtes auf Religionsfreiheit existiert und alle diese Forderungen von eben diesem geschützt sind, verursacht die Einforderung dieser Rechte Misstrauen, emotionale Ablehnung, Skepsis oder sogar Feindseligkeit. Ambivalenzen treten selbstverständlich auch auf der Seite der Minderheiten zum Vorschein: Zwar kommt es einerseits zur aktiven Forderung nach mehr Repräsentation, nach Identitätspolitik sowie der Etablierung von Quotenregelungen und Antidiskriminierungsgesetzen, auf der anderen Seite existiert aber auch die Sehnsucht nach dem Auflösen jener Kategorien, die ethnische Hintergründe hervorheben (Supik 2014). Ablehnung und Akzeptanz können daher gleichzeitig existieren: Auch wenn Pluralität und Heterogenität kognitiv akzeptiert und auf konstitutioneller Ebene von Werten der Gleichheit als Normalität empfunden werden, können fundamentale Grundrechte emotional abgelehnt werden, wenn sie von Minderheiten beansprucht werden. Diese Dissonanz zwischen kognitiver Akzeptanz und emotionaler Distanz schafft konflikthafte Ambivalenzen und Unklarheiten in postmigrantischen Gesellschaften (Foroutan/Canan 2016).

4. Allianzen: Die migrantische Realität von Gesellschaften führt dazu, dass auf persönlicher, beruflicher und sozialer Ebene Beziehungen entstehen, die Menschen mit verschiedenen Hintergründen und Erfahrungen miteinander verbinden. Über familiäre Verflechtungen, Freundeskreise, schulische und berufliche Ausbildung, durch Vereine, Gewerkschaften und ver- 
schiedene Formen des zivilgesellschaftlichen Engagements entfalten und verbreiten sich neue Arten von Wissen. Diese neuen Verflechtungen können Empathie erzeugen und politische Einstellungen hervorbringen, auf deren Grundlage sogenannte postmigrantische Allianzen entstehen. Diese definieren sich nicht mehr über migrantische Biografien, Nationalität oder Religionszugehörigkeit, sondern über eine geteilte Haltung die auf Gleichheit, pluraler Demokratie und der aktiven Akzeptanz von Diversität und Vielfalt beruht (Foroutan 2016: 228). MigrantInnen und ihre Nachkommen kämpfen nicht alleine für die Umsetzung des Gleichheitsanspruches, sondern gemeinsam mit Gleichgesinnten und Verbündeten. Postmigrantische Allianzen sind ein mächtiges Instrument, um diskriminierende Strukturen herauszufordern und anzufechten: Sie ermöglichen einen gemeinsamen Kampf gegen rassistische Haltungen und die Stigmatisierung von MigrantInnen, der nicht von paternalistischen Strukturen, sondern von einer Zusammenarbeit auf Augenhöhe gekennzeichnet ist. Die postmigrantische Perspektive eröffnet damit einen Blick auf neue Beziehungen, die abgesehen von homogenen Peergroups unterschiedliche Menschen im gemeinsamen Kampf verbinden und auf der Basis der geteilten Haltung zusammenbringen (Foroutan 2015: 18; Broden/Mecheril 2014: 15). Die gemeinsame Fokussierung auf eine Agenda, geteilte Werte und Solidarität untereinander wirken als Bindeglieder (Parsons 1967: 704; Sabatier 1993: 21). Empathie und emotionale Verbundenheit dienen als Kitt dieser Zusammenschlüsse und fördern das Eintreten für Diversität und Vielfalt mit dem Ziel, sozialen Wandel zu gestalten (Nussbaum 1997: 90). Aber auch unabhängig von persönlichen Beziehungen und Interaktionen kann es zu Allianzbildungen kommen. Auch strategisch konkrete Ziele, wie das Erreichen konkreter Inhalte, Gesetze oder der Widerstand gegen konkrete Bewegungen, können als Verbindung innerhalb der Zusammenschlüsse dienen, solange es um geteilte Ziele und Visionen in Bezug auf die Umsetzung des Versprechens der pluralen Demokratie, Heterogenität und Vielfalt geht. Allianzen strukturieren Identitätskonzepte und Konzepte der Zugehörigkeit neu, die als Absage an Verbundenheit durch geteilte Ethnizität, Herkunft oder Homogenität im Allgemeinen verstanden werden können (Bauman 1992), und schaffen eine Form der hybriden Peergroup-Identität, die in erster Linie auf eine geteilte Haltung zurückzuführen ist (Brah et al. 2005).

5. Antagonismen: Postmigrantischen Gesellschaften ist ein signifikantes Konfliktpotenzial inhärent. Die zunehmende Hybridisierung und Heterogenisierung der Gesellschaft stößt bei weiten Teilen der Mehrheitsbevölkerung auf Ablehnung. Im Gegensatz zu postmigrantischen Allianzen bilden sich daher auch Gruppen mit antagonistischen Positionen zu Migration und sämtlichen Begleitphänomenen. Diese Positionierungen, die gekennzeichnet sind durch negative Einstellungen gegenüber einer auf dem demo- 
kratischen Gleichheitsgrundsatz beruhenden Ausweitung von materieller und symbolischer Zugehörigkeit, kämpfen um politische Dominanz und ihre Vormachtstellung, siehe etwa den Slogan des französischen rechtsextremen Front National: »Les Francais d'abord «(Laurence/Goodliffe 2013: 36). Der Konflikt zwischen Gruppen, die Pluralität für wünschenswert erachten und jenen, die sich Homogenität und Eindeutigkeit herbeisehnen, führt zunehmend zu sichtbaren und spürbaren Positionen innerhalb von postmigrantischen Gesellschaften. Der Dualismus zwischen BefürworterInnen und GegnerInnen dominiert die gesellschaftspolitische Agenda und führt zu neuen binären Konfrontationen entlang der Konfliktlinie Migration.

Während die eine Seite die migrantische Realität in der Gesellschaft nicht lediglich akzeptiert, sondern sogar als wünschenswert oder bereits als Normalität erachtet, steht die andere Seite für eine Gesellschaft auf der Grundlage eindeutiger sozialer Ordnung ein, die sich durch exklusiven Nationalismus und ethnische Homogenität charakterisiert. Auch wenn es diese gesellschaftlichen und politischen Gegensätze schon vor der offiziellen Bestätigung, Einwanderungsland geworden zu sein, gegeben hat, so erreichen sie in postmigrantischen Gesellschaften eine weitere Dimension: Die rechtliche und moralische Legitimität der Aushandlung von Gleichheit begünstigt zwar die Etablierung einer moralischen Mehrheit, führt aber auch dazu, dass die antagonistische Minderheit sich umso vehementer gegen diese Entwicklungen zur Wehr setzt. Minderheitenrechte werden offener angegriffen oder angezweifelt und Überzeugungen, Zugehörigkeit und Privilegien ununterbrochen neu ausgehandelt (Foroutan 2016: 241). Der Kampf um Ressourcen und die Aushandlungen über nationale Identität intensivieren sowohl die Debatten über die Ankunft von MigrantInnen als auch über ihre soziale Stellung als AußenseiterInnen gegenüber etablierten Akteuren (Elias/Scotson 2002: 7).

Zwischen diesen beiden gegensätzlichen Positionen besteht eine große unentschlossene Mitte, die in beide Richtungen mobilisierbar zu sein scheint. Im Kampf um Zulauf und Unterstützung aus diesen Kreisen bedienen sich beide Lager wirtschaftlicher und demografischer Argumente. In Ländern wie Frankreich, Dänemark, Großbritannien, Ungarn und Polen, in denen rechtspopulistische Parteien in den letzten Wahlen mitunter zwischen 25 und 50 Prozent der Stimmen erhielten, wurde die mobilisierbare Mitte mit ebendiesen Argumenten zu wirtschaftlicher Entwicklung, demografischem Wandel und Themen des Arbeitsmarktes zu überzeugen versucht. Rechtspopulismus und die Neigung zu rechtsextremen Positionen kann jedoch nicht lediglich mit der Angst vor wirtschaftlichem Abstieg erklärt werden. Selbst wohlhabende europäische Volkswirtschaften wie Österreich, die Schweiz oder die skandinavischen Länder verzeichnen einen starken 
Aufstieg rechtspopulistischer Parteien. Es scheint einen sehr fruchtbaren Boden für extremistische und menschenfeindliche Stimmungen zu geben, der es leichtmacht, Menschen in Richtung antagonistischer Positionen zu mobilisieren, und der andere Parteien in diesem Zuge dazu drängt, sich bezüglich ihrer Standpunkte zu Migration und Vielfalt mehr nach rechts $\mathrm{zu}$ verschieben, um keine Wählerstimmen einzubüßen (Mudde 2007).

\section{SCHLUSSFOLGERUNGEN}

Die postmigrantische Gesellschaft ist von Ambivalenzen und Unübersichtlichkeit geprägt, was sie konfliktreich macht; gleichzeitig beinhaltet sie das Versprechen einer radikalen, über das Migrantische hinausweisenden Utopie der Gleichheit, die außerhalb der Herkunft verhandelt wird. In dem Begriff steckt gleichzeitig ein normsetzender Verweis auf eine gesellschaftspolitisch anzustrebende Entwicklung, nicht nur eine analytisch klar umgrenzte Kategorie derzeitiger Gesellschaft. Zusammenfassend kann also festgehalten werden, dass postmigrantische Gesellschaften zwar von einer Utopie der Gleichheit angetrieben werden, da diese jedoch unverwirklicht bleibt, sind sie geprägt von einer permanenten Ambivalenz, die eine Polarisierung zwischen MigrationsbefürworterInnen und MigrationsgegnerInnen erzeugt. Dabei steht Migration als Chiffre für Pluralität und die in der pluralen Demokratie stattfindenden fundamentalen Aushandlungen von Rechten, Anerkennung und Chancengleichheit sowie von Teilhabe und Zugehörigkeit in der Gesellschaft.

Die postmigrantische Perspektive fordert dazu auf, den Blick auf das Versprechen der pluralen Demokratie zu lenken, die ihr Pluralitätsbekenntnis in dem Selbstbild, ein Einwanderungsland zu sein, festschreibt. Die Aushandlungen, die hierbei erfolgen, richten sich gegen Strukturen und Prozesse der Ungleichheit, gegen Diskurse der Kulturalisierung und Ethnisierung sowie gegen Diskriminierung und Rassismus. Die postmigrantische Vision umfasst in diesem Zusammenhang ein neues, wirklich plurales Gesellschaftsverständnis.

\section{LITERATUR}

Bauman, Zygmunt 1992: Soil, Blood and Identity, in: The Sociological Review 40(4), 675-701.

Brah, Avtar/Coombes, Annie 2005: Hybridity and its Discontents: Politics, Science, Culture. London.

Broden, Anne/Mecheril, Paul 2014: Solidarität in der Migrationsgesellschaft. Einleitende Bemerkungen, in: Anne Broden/Paul Mecheril (Hg.): Solidari- 
tät in der Migrationsgesellschaft. Befragung einer normativen Grundlage. Bielefeld, 7-20.

Elias, Norbert/Scotson, John L. 2002: Etablierte und Außenseiter. Frankfurt a.M.

Foroutan, Naika/Canan, Coşkun/Beigang, Steffen/Arnold, Sina/Schwarze Benjamin/Kalkum, Dorina 2014: Deutschland postmigrantisch I: Gesellschaft, Religion, Identität, Berlin.

Foroutan, Naika 2015: Konviviale Integration in postmigrantischen Gesellschaften, in: Frank Adloff/Volker M. Heins (Hg.): Konvivialismus. Eine Debatte. Bielefeld, 205-216.

Foroutan, Naika 2016: Postmigrantische Gesellschaften, in: Heinz Ulrich Brinkmann/Martina Sauer (Hg.): Einwanderungsgesellschaft Deutschland. Entwicklung und Stand der Integration. Wiesbaden, 227-254.

Foroutan, Naika/Canan, Coşkun 2016: The Paradox of Equal Belonging of Muslims, in: Islamophobia Studies Journal 3 (2), 159-176.

Langhoff, Shermin 2011: Die Herkunft spielt keine Rolle-» Postmigrantisches » Theater im Ballhaus Naunynstraße, Bundeszentrale für politische Bildung, www.bpb.de/gesellschaft/kultur/kulturelle-bildung/60135/interviewmit-shermin-langhoff. (abgerufen am 03.07.2017).

Laurence, Jonathan/Goodliffe, Gabriel 2013: The French Debate on National Identity and the Sarkozy Presidency: A Retrospective, in: The International Spectator, 48:1, 34-47.

Mudde, Cas 2007: Populist Radical Right Parties in Europe. Cambridge.

Nienhaus, Lisa 2015: Ostdeutsch, männlich, pessimistisch. Frankfurter Allgemeine Zeitung, www.faz.net/aktuell/politik/inland/afd-ostdeutsch-maenn lich-pessimistisch-13924829.html (abgerufen am 03.07.2017).

Nussbaum, Martha C. 1997: Cultivating Humanity. A Classical Defense of Reform in Liberal Education. Cambridge.

Parsons, Talcott 1967: The Structure of Social Action. New York.

Sabatier, Paul A. 1993: Policy Change over a Decade or More, in: Paul A. Sabatier/Hank C. Jenkins-Smith (Hg.): Policy Change and Learning: An Advocacy Coalition Approach. Boulder, 13-39.

Spielhaus, Riem 2011: Wer ist hier Muslim? Die Entwicklung eines islamischen Bewusstseins in Deutschland zwischen Selbstidentifikation und Fremdzuschreibung. Würzburg.

Spielhaus, Riem 2012: Studien in der postmigrantischen Gesellschaft: Eine kritische Auseinandersetzung, in: Kongressdokumentation Bundesfachkongress Interkultur 2012, 96-100.

Spivak, Gayatri Chakravorty 1988: Can the Subaltern Speak?, in: Cary Nelson/ Lawrence Grossberg (Hg.): Marxism and the Interpretation of Culture. Chicago, 271-313. 
Supik, Linda 2014: Statistik und Rassismus. Das Dilemma der Erfassung von Ethnizität. Frankfurt a.M.

Unabhängige Kommission »Zuwanderung« 2001: Zuwanderung gestalten Integration fördern. Bericht der Unabhängigen Kommission »Zuwanderung«. Berlin. 
